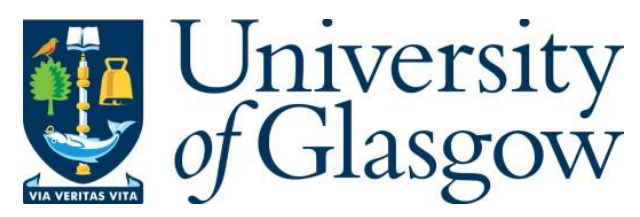

Loram, I., Cunningham, R., Zenzeri, J. and Gollee, H. (2016) Intermittent Control of Unstable Multivariate Systems with Uncertain System Parameters. In: IEEE 38th Annual International Conference of the Engineering in Medicine and Biology Society (EMBC), Orlando, FL, USA, 16-20 Aug 2016, pp. 17-20. ISBN 9781457702198.

There may be differences between this version and the published version. You are advised to consult the publisher's version if you wish to cite from it.

$\underline{\text { http://eprints.gla.ac.uk/144999/ }}$

Deposited on: 1 August 2017

Enlighten - Research publications by members of the University of Glasgow http://eprints.gla.ac.uk 


\title{
Intermittent control of unstable multivariate systems with uncertain system parameters*
}

\author{
I. Loram ${ }^{1}$, R. Cunningham ${ }^{1}$, J. Zenzeri ${ }^{2}$ and H. Gollee ${ }^{3}$
}

\begin{abstract}
Multivariable intermittent control (MIC) combines stability with flexibility in the control of unstable systems. Using an underlying continuous-time optimal control design, MIC uses models of the physical system to generate multivariate open-loop control signals between samples of the observed state. Using accurate model values of physical system parameters, stability of the closed loop system is not dependent upon sample interval.

Here we consider the sensitivity of MIC to inaccurate model values of system parameters. The high dimensionality of multiple parameters combined with an unstable open loop system ensures the ratio of hyper-volumes containing good to bad parameter combinations resembles a "needle in a haystack". Is this sensitivity a problem or an asset?

Prediction error between open loop and observed states provides the basis for triggering a sampling event but is also sensitive to inaccurate model values. Investigation of the mapping between prediction error and model values of physical parameters illustrates the value of prediction error to identify combinations of parameters giving stable closed loop control with low state error, similar to that provided by accurate values.

Sensitivity of prediction error to model inaccuracy is potentially an asset facilitating adaptation and supporting the rationale for MIC to combine control with flexibility.
\end{abstract}

\section{INTRODUCTION}

Adaptive control must address the dual demands of regulating motor control and learning new motor behaviour. There is a tension between the dual demands of exploitation and exploration, or alternatively between stability and plasticity. This tension is maximised when the controlled system is unstable, such as the multi-link mechanical structure of the upright human. An unstable mechanical structure benefits from a high control bandwidth. If regulation and stability is too good, the sensorimotor signals have low variability and bandwidth which compromises effective learning and adaptation. Vertebrate species operate successfully in a variety of environmental niches. However, if "success" depends upon adaptability and flexibility, then regulation and stability need only be minimally sufficient, while biological priority is given to a high bandwidth of decision making. Maximising the bandwidth of state dependent motor decision making requires that selection and optimisation of control structure and design are implemented within the sensorimotor feedback loop $[1,2]$. An on-board, bio-inspired architecture suitable for adaptable robots operating in complex, changing environments, for example operating safely in close physical proximity with humans,

*Supported by EPSRC EP/F068514/1, EP/F069022/1, EP/F06974X/1.

${ }^{1}$ School of Healthcare Science, Manchester Metropolitan University, Manchester, UK; email: i.loram@mmu.ac.uk,

Ryan.cunningham@mmu.ac.uk should maximise the decision making bandwidth and should implement state dependent selection and optimisation within the feedback loop.

Investigation from many authors has considered whether human sensorimotor control is intermittent. In short, intermittent v. continuous control trades state dependent online flexibility for control bandwidth [2]. The intermittent rather than continuous use of sensory information to update control signals implies an event trigger determining when to use sensory information, a discrete sampling/initialisation process and a hold process constructing a time varying control trajectory (Fig. 1), [3]. The duration for which control is open loop is called the open loop interval. A disadvantage of intermittent control (IC) is the reduction in control bandwidth. Benefits are (i) the event related possibility to iteratively reinitialise the control basis and (ii) the availability of predictively stabilised time for state dependent selection and optimisation $[1,2,4]$.

Recent theoretical and methodological advances have provided new experimental evidence that while human sustained tracking masquerades as continuous control, humans actually uses sensory feedback intermittently to sequentially update intervals of open loop predictive control $[2,5,6]$. An attribute of open loop predictive control of unstable systems, is that control is sensitive to inaccurate model values of physical system parameters. While sensitivity presents a challenge to control of unstable systems, it also presents possible advantages when control needs to be adaptable.

Within IC, prediction error is used to trigger the intermittent use of sensory feedback (Fig 1). Here, our question is whether prediction error provides the information required to identify good model parameter values, giving stable closed loop control similar to that obtained with the accurate values.

\section{Methods}

Multivariate intermittent control (MIC) is based on the continuous-time optimal control design method using the observer, predictor state-feedback architecture [3, 7]. MIC uses an event detection mechanism. A system matched hold, using the underlying continuous optimal control design, generates multivariate open-loop control signals between samples of the predicted state. This serial, sequential process provides a single channel of control with optimised sensor fusion and motor synergies [3].

\footnotetext{
${ }^{2}$ Robotics, Brain and Cognitive Sciences Dept., Istituto Italiano di Tecnologia, Genoa, Italy; email jacopo.zenzeri@iit.it

${ }^{3}$ School of Engineering, University of Glasgow, Glasgow, UK; email henrik.gollee@glasgow.ac.uk
} 
The system-matched hold (SMH) is the key component of the intermittent control. As described previously [7], the SMH state $\hat{x}_{h}$ evolves in a local intermittent time frame $\tau$ as

$$
\begin{aligned}
& d / d t \hat{x}_{h}(\tau)=A_{h} \hat{x}_{h}(\tau) \\
& \text { where } A_{h}=A_{c} \text { and } \hat{x}_{h}(0)=\hat{x}_{p}\left(t_{i}-t_{d}\right)
\end{aligned}
$$

where $\mathbf{A}_{c}$ is the closed-loop system matrix of the equivalent continuous system, and $\hat{x}_{p}$ is the predicted state. The hold state $\hat{x}_{h}$ replaces the predictor state $\hat{x}_{p}$ in the controller equation. Other holds (where $\mathbf{A}_{h} \neq \mathbf{A}_{c}$ ) are possible.

The intermittent controller generates an open loop control signal based on the hold state $\hat{x}_{h}$ (1). At the discrete intermittent sample times $t_{i}$, the hold state is reset to the estimated system state $\hat{x}_{w}$ generated by the observer, thus feedback occurs at the intermittent sample times $t_{i}$. The sample times are constrained to be at least $\Delta_{\min }$ apart. But, in addition to this constraint, feedback only takes place when it is needed. Such feedback is required when the open-loop hold state $\hat{x}_{h}$ differs significantly from the closed-loop observer state $\hat{x}_{0}{ }^{w}$ indicating normally, the presence of disturbances. There are many ways to measure such a discrepancy. Following [7,8], we compute a quadratic function of the prediction error $\mathbf{e}_{h p}$, such that $\mathrm{E}>1$ triggers sampling of the observed state $x_{o}{ }^{w}$,

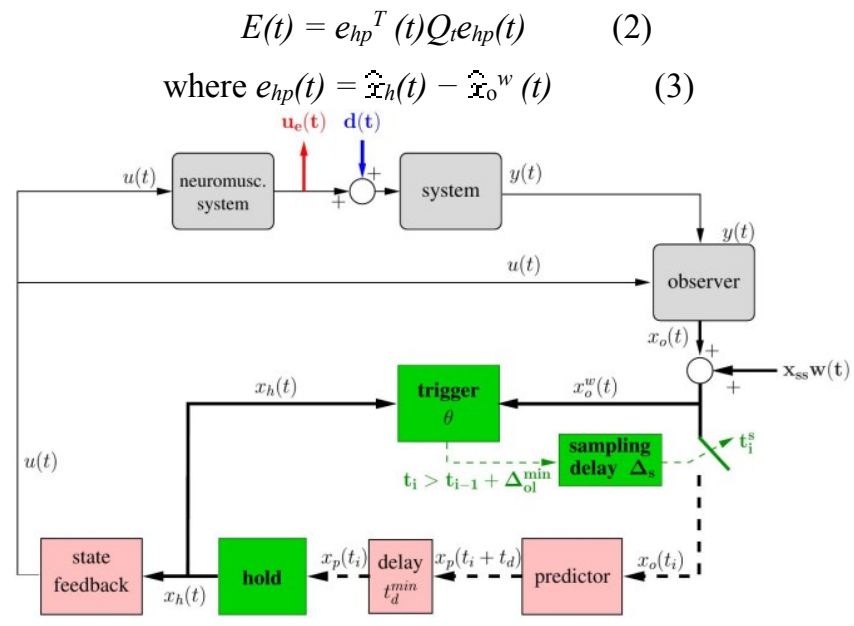

Fig. 1. Implementation of intermittent control. In common with continuous optimal control, the observer and predictor contain models reproducing the behaviour of the physical system. The command signal, $u$, serves as a single input to the "Plant" whose neuro-muscular system synergistically translates it to the multiple system segments. By comparison with continuous control, intermittent control, includes the additional processes (green) of event trigger, sampling, and generalised hold [3].

The hold generates open loop control signals equal to the equivalent underlying continuous control in the absence of disturbances $d$ and changes in set point $w$.

Prediction error is the difference between the predicted hold state $x_{h}$ and observed state $x_{o}{ }^{w}$. Following a specified minimum open loop interval, sampling of the observed state to update open loop control is triggered by prediction error exceeding a threshold.

Here the observer, predictor and hold use values of physical system parameters (segmental mass $m$, length $l$, mass location $c$, radius of gyration $r$ ) which are not known accurately. For all simulations the delay, sampling delay and minimum open loop interval were $0.2,0$ and $0.1 \mathrm{~s}$ respectively. The neuromuscular system was second order with two time constants of $0.1 \mathrm{~s}$. where $\boldsymbol{Q}_{t}$ is a positive semi-definite matrix representing appropriate weights for each state [8]. $\mathrm{E}$ is thus a continuously evolving scalar norm of the prediction error $\mathbf{e}_{h p}$.

The unstable, mechanical system comprises a linearized, sagittal (2D) version of the three link (lower leg, upper leg, trunk-arms-head), three joint (ankle, knee, hip) model of human posture. For this system each segment has four physical parameters: mass $(m)$, link length $(l)$, CoM location $(c)$, and radius of gyration $(r)$ with values taken from Figure 4.1 and Table 4.1 of [9]. The controller is designed using linear quadratic regulation as described previously $[3,8]$.

Previously, the observer and predictor used exact values of physical parameters within the equations modelling the system. Here we used inexact model values expressed relative to the true value. To provide a measure of closed loop stability, we calculated the maximum real pole of the underlying continuous closed loop system of the SMH.

Using the set of model parameter values as a multidimensional input $(X)$ we simulated, repeatedly, ten seconds of movement generated by a change in set point $w$ of size $w_{0}$ (Fig. 2). Since prediction error $(E)$ varies continuous (eq. (2)), we calculated a scalar measure of prediction error $(\bar{e})$, normalised for size of movement:

$$
\bar{e}=-\log _{10}\left(\bar{E} / w_{0}\right)
$$

where $\bar{E}$ is the mean of $\mathrm{E}$ for times $3<\mathrm{t} \leq 10$ s. We also calculated State Error, defined as the root mean square value
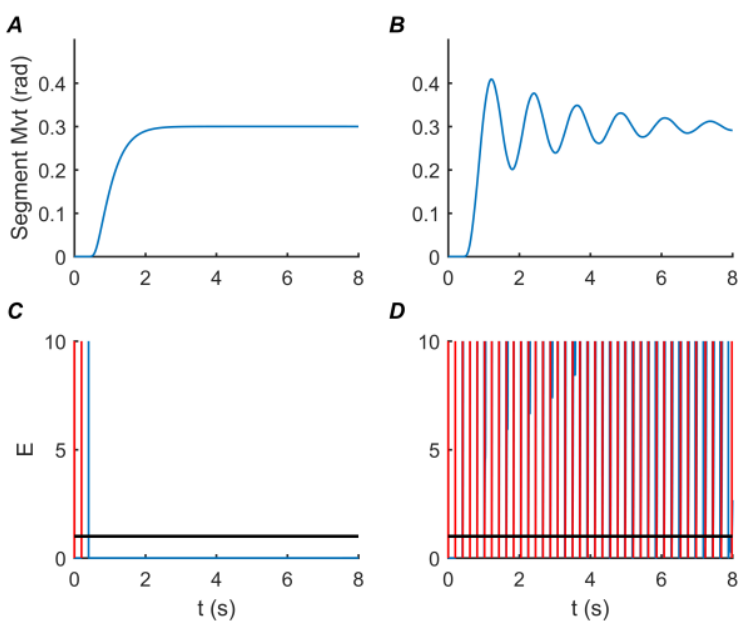

Figure 2: Representative effect of inaccurate controller model on movement of single segment following a change in set point. $\boldsymbol{A}, \boldsymbol{B}$ : segment angle (radians). $\boldsymbol{C}, \boldsymbol{D}$ : Prediction Error (blue). Trigger times (red).

Left: controller uses correct values of physical system parameters. Here, in the absence of disturbance, following triggering of the change in set point at $0.2 \mathrm{~s}$, (vertical red line), and following a delay of $0.2 \mathrm{~s}$, the prediction error (blue) is reduced to zero by accurate control action which remains open loop for the duration of the movement.

Right controller uses incorrect value of system mass ( $1.4 \mathrm{x}$ correct value). Although open loop control is inaccurate, causing rapidly rising prediction error, repeated triggering and increased oscillation, the closed loop system is stable since the oscillations decay. N.B. IC adapts naturally to inaccurate parameter values, measurement noise and disturbances by the trigger mechanism which promotes more frequent use of sensory feedback. 
of the position component of the hold state $x_{h}$ for the same 7 seconds.

Our objective was to map the relationship between model values of system parameters $(\mathrm{X})$ and normalised prediction error $(\bar{e})$ and consider the value of that mapping for adapting control.

To provide a uniform, economical selection of values for mapping, we sampled randomly, uniformly within a multidimensional convex hull, initialised on the vertices of a hypercube, at $\pm 40 \%$ of the true parameter values. Following 800 trials, the convex hull was contracted to surround the cases providing the lowest 200 values of prediction error $(\bar{e})$. To prevent shrinking to an unrepresentative local minimum, every other trial sampled, randomly, normally (S.D. $=0.1$ ), a location outside a randomly chosen vertex of the convex hull. After each iteration of 200 trials, the convex hull was recalculated.

To provide a, smooth representation, robust to a variety of distributions, we modelled the mapping $\mathrm{X}$ to $\mathrm{y}$ as a multidimensional Gaussian process [10]. We used a squared exponential covariance function with Automatic Relevance Determination providing individual scaling for each dimension, and a second order polynomial mean function. Using the Gaussian process model to generate values at test locations, we performed a global pattern search, constrained within the convex hull to locate the parameter values of the minimum prediction error.

To regulate the scale of the multidimensional mapping, we adopted a strategy of first investigating the four parameters of the upper link, with the lower segments immobilised. This approach is to optimise values of the upper link until they are reliable enough to be used as fixed parameters while mapping parameter values to prediction
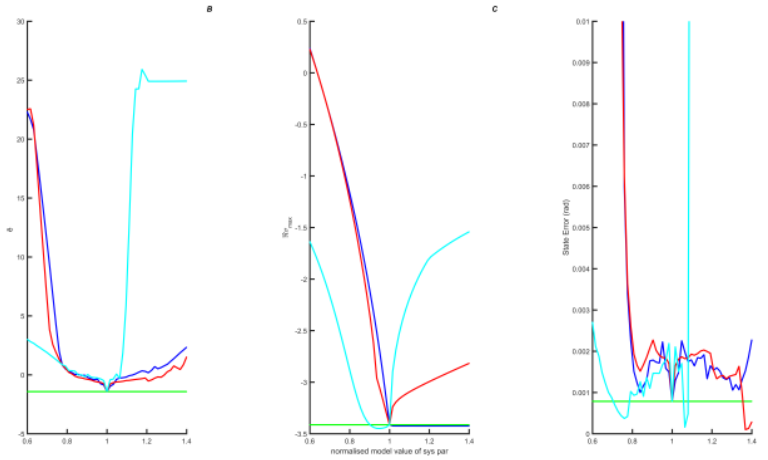

Fig. 3: Univariate mapping of prediction error, stability and performance to normalised model values of physical system parameters. These relationships were produced using movement of a single (upper) segment following a change in set point, as shown in Fig 2. $\boldsymbol{A}$. Prediction error $(\bar{e})$. B . Maximum real pole of the continuous close loop system underlying the system matched hold. C. State error, rms value of the final 7 seconds.

The true value of the physical parameter is easily identified from its univariate variation with prediction error. IC is stable for a variety of model values.

Inaccurate model values cause Prediction error to increase even when stability is unchanging (c.f. $m_{l}$ ). Decreased closed loop stability amplifies the growth of prediction error.

Prediction error is related to State error, so regulation of parameters to minimise prediction error would also provide good control. Compared with prediction error, State error but provides a less useful identification of physical parameter values. error for the next lower segment. This sequential method of mapping parameters reduces the problem to a size that does not scale with the number of dimensions and is inspired by the observation that humans learn head control, followed by trunk control progressively down trunk segments until control of the head and complete trunk is sufficient to enable free sitting and subsequently standing balance [11].
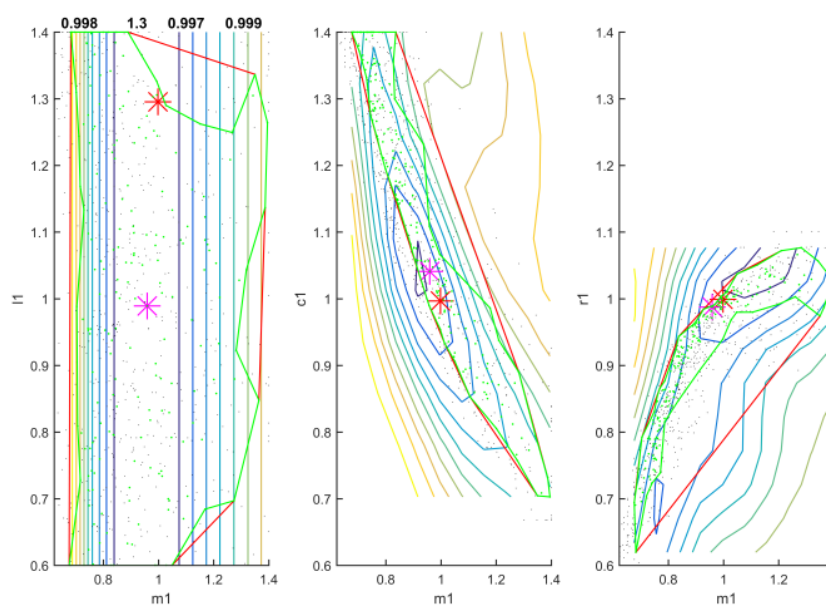

Figure 4 Covariation of prediction error with model parameters. Data from movement of single, upper segment following change in set point. Contours show prediction error $(\bar{e})$ related to pairs of parameter values. Red lines show convex hull. Green lines show convex hull shrunk to data points. Green and black dots show samples giving lowest 800 and 200 prediction errors. Red star, Gaussian process minimum. Magenta star lowest data value.

The convex hull defines combinations of parameters giving minimal prediction error.

\section{RESULTS}

When model parameters were varied individually, prediction error $(\bar{e})$ increased with parameter inaccuracy allowing easy identification of true physical values (Fig 3). Inaccurate parameter values reduce the stability of the underlying closed loop system, but the underlying closed loop system remains stable for a range of inaccurate parameter values. Inaccurate parameter values increase state error, although the relationship is not as well defined as that between prediction error and parameter values.

Figs. 4 and 5 show that covariation of model parameters led to more complicated relationships between input parameter values and prediction error. However, the multivariate mapping of prediction error to model values allows effective identification of combinations of parameters giving stable intermittent control with minimal prediction error, and with minimal state error (Fig. 5). Figure 5 shows that reducing state errors to acceptably low levels requires close regulation of model parameter values within narrow limits. Some parameters (e.g. mass of the upper segment, and length of the middle segment), and or parameter combinations require closer regulation than others.

Sequential application of the search to model parameters for upper (Fig 4), middle (Fig 5) and lower segments, 4000 trials at each stage, and the Gaussian process model, led to 12 parameter values (below) giving a prediction error, maximum 
real pole and state error of $0.98,-2.4,0.025$ rads, compared with $0.16,-2.6,0.007$ rads for true values.

\begin{tabular}{|l|llllllllllll|}
\hline Quantity & $\mathrm{m}_{1}$ & $\mathrm{l}_{1}$ & $\mathrm{c}_{1}$ & $\mathrm{r}_{1}$ & $\mathrm{~m}_{2}$ & $\mathrm{l}_{2}$ & $\mathrm{r}_{2}$ & $\mathrm{c}_{2}$ & $\mathrm{~m}_{3}$ & $\mathrm{l}_{3}$ & $\mathrm{c}_{3}$ & $\mathrm{r}_{3}$ \\
\hline Estimated & 1.01 & 0.88 & 1.00 & 1.01 & 1.15 & 1.00 & 0.80 & 1.00 & 0.7 & 1.00 & 0.7 & 1.3 \\
\hline $\pm 95 \% \mathrm{ci}$ & 0.03 & 11 & 0.04 & 0.5 & 0.02 & 0.005 & 0.03 & 0.7 & 0.04 & 0.1 & 0.4 & 0.02 \\
\hline
\end{tabular}
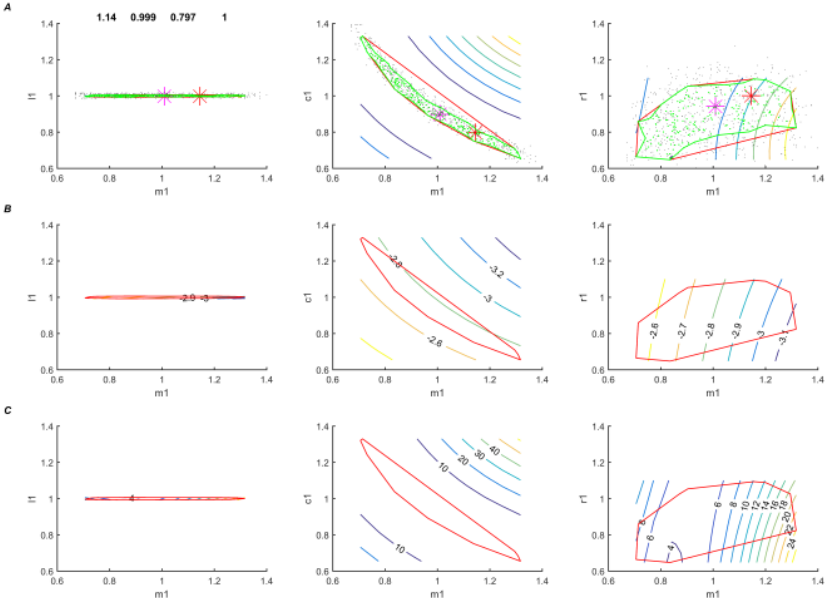

Figure 5 Covariation of prediction error, stability and performance with model parameters. Data from movement of upper two segments following change in set point. Model values of upper segment were fixed using the combination identified previously (Fig. 4).

Panels A: Contours show prediction error $(\bar{e})$ related to pairs of parameter values. Red lines show convex hull. Green lines show convex hull shrunk to data points. Green and black dots show samples giving lowest 800 and 200 prediction errors. Red star, Gaussian process minimum. Magenta star lowest data value.

Panels B: Contours show maximum real pole of underlying closed loop system (SMH). Panels $\boldsymbol{C}$. Contours show state error (radians).

Within the convex hull region, prediction error defines combinations of parameters giving minimal prediction error. Minimal prediction error is related to maximal close loop stability and minimum state error.

\section{CONCLUSIONS}

MIC can stabilise an unstable multi-link mechanical system representing the upright adult human. However, control is sensitive to inaccurate model values of the physical system. From the evidence we formulate the following hypotheses:

- Prediction error provides sufficient information to identify combinations of parameter values giving stable intermittent control with minimal state error close to that obtained with true values.

- Top down, sequential, identification of segments simplifies identification of the multilink system.

MIC operates intermittent sampling of sensory feedback to update predictive open loop control. Inaccurate model values ensures the observer and predictor insert error, disturbing the closed loop system. This error is amplified by inaccurate open loop control when the physical system is unstable. This sensitivity of MIC to inaccurate model values is compounded by the number of inaccurate parameters. Clearly the unique accurate parameter values are present within the set of all possible values, however, the number of values giving unstable or poor control multiplies with the number of parameters. Consequently the region of parameter combinations producing low state error is rather small requiring close regulation of model parameter values (Fig 5).

The sensitivity of prediction error to inaccurate model values is a potential advantage providing an error signal enabling adaptation of control. Identification of the true physical parameters is unnecessary providing it is possible to identify combinations of parameters giving good, stable control [12] Our exploration of the mapping between parameter values, and prediction error suggests the identification of combinations of parameters giving good control is tractable using prediction error. It appears that minimisation of prediction error will also minimise state error. This would ensure that the dual control of parameters and states resolves into a single process. If prediction error generated naturally within MIC enables identification of good model parameters, this sensitivity to model inaccuracy becomes an asset which facilitates adaptation and which supports the combination of control with flexibility.

\section{ACKNOWLEDGMENT}

The authors gratefully acknowledge discussion with and feedback from Prof Peter J Gawthrop.

\section{REFERENCES}

[1] E. Ronco, T. Arsan, and P. J. Gawthrop, "Open-loop intermittent feedback control: practical continuous-time GPC," Control Theory and Applications, IEE Proceedings -, vol. 146, pp. 426434, 1999.

[2] I. D. Loram, C. van de Kamp, M. Lakie, H. Gollee, and P. J. Gawthrop, "Does the motor system need intermittent control?," Exerc Sport Sci Rev, vol. 42, pp. 117-25, Jul 2014.

[3] P. Gawthrop, H. Gollee, and I. Loram, "Intermittent Control in Man and Machine," in Event-based control and signal processing, ed: CRC Press/Taylor \& Francis, 2015 (Nov), p. http://arxiv.org/abs/1407.3543.

P. Gawthrop, D. Wagg, S. Neild, and L. Wang, "Powerconstrained intermittent control," International Journal of Control, vol. 86, pp. 396-409, Mar 12013.

[5] C. van de Kamp, P. J. Gawthrop, H. Gollee, and I. D. Loram, "Refractoriness in sustained visuo-manual control: is the refractory duration intrinsic or does it depend on external system properties?," PLoS Comput Biol, vol. 9, p. e1002843, 2013.

[6] I. D. Loram, C. van de Kamp, H. Gollee, and P. J. Gawthrop, "Identification of intermittent control in man and machine," $J R$ Soc Interface, vol. 9, pp. 2070-84, Sep 72012.

[7] P. Gawthrop, I. Loram, M. Lakie, and H. Gollee, "Intermittent control: a computational theory of human control," Biol Cybern, vol. 104, pp. 31-51, Feb 2011.

[8] I. Loram, P. Gawthrop, and H. Gollee, "Intermittent control of unstable multivariate systems," in Engineering in Medicine and Biology Society (EMBC), 2015 , pp. 1436-1439.

[9] D. A. Winter, Biomechanics and Motor Control of Human Movement: John Wiley \& Sons, Inc., 2009.

[10] C. E. Rasmussen and C. K. I. Williams, Gaussian Processes for Machine Learning (Adaptive Computation and Machine Learning): The MIT Press, 2005.

[11] P. Butler, M. S. Saavedra, M. M. Sofranac, M. S. Jarvis, and M. Woollacott, "Refinement, reliability and validity of the segmental assessment of trunk control (SATCo)," Pediatric physical therapy: the official publication of the Section on Pediatrics of the American Physical Therapy Association, vol. 22, p. 246, 2010.

[12] R. N. Gutenkunst, J. J. Waterfall, F. P. Casey, K. S. Brown, C. R. Myers, and J. P. Sethna, "Universally sloppy parameter sensitivities in systems biology models," PLoS Comput Biol, vol. 3, pp. 1871-78, Oct 2007. 